\title{
Place of Perineal Rehabilitation in the Management of Female Stress Urinary Incontinence
}

\author{
Davelle Doungou Ngandzali ${ }^{1,2^{*}}$, Hasna Ouazzani ${ }^{1}$, Amour Parfait Emmanuel Ngouma Youmbert ${ }^{2}$, Yvette Moigny Gaju ${ }^{2}$, \\ Youness Abdelfettah ${ }^{1}$ \\ ${ }^{1}$ Department of Physical and Rehabilitation Medicine, University Hospital Mohammed VI Marrakesh; Faculty of Medicine, Cadi Ayad \\ University Marrakesh; Morocco \\ ${ }^{2}$ Department of Functional Rehabilitation, University Hospital of Brazzaville; Faculty of Medicine, Marien Ngouabi University \\ Brazzaville; Congo
}

\author{
DOI: $10.36348 /$ sijog.2021.v04i04.013 \\ | Received: 14.03.2021 | Accepted: 20.04.2021 | Published: 25.04 .2021 \\ *Corresponding author: Dr. Davelle Doungou Ngandzali
}

\section{Abstract}

Introduction: Female stress urinary incontinence is a common affection that affects women in the general population. It can be improved by non-invasive medical techniques. Aims: To determine the value of perineal rehabilitation in the treatment of female stress urinary incontinence through the experience of the Department of Physical Medicine and Rehabilitation CHU Med VI of Marrakech. Patients and methods: This is a prospective, monocentric study that included 10 patients with stress urinary incontinence. They benefited from a multimodal rehabilitation protocol carried out in a minimum of 10 sessions. Results: The muscle tone of the pubococcygeal bundles of the elevator muscles of the anus was significantly improved after rehabilitation. The improvement in tone was correlated with the reduction in the frequency of leaks. The subjective satisfaction rate reached $80 \%$. Conclusion: Perineal rehabilitation appears from the experience of our department to be effective for the treatment of stress urinary incontinence. It should be offered as a first-line option.

Keywords: Stress urinary incontinence; Perineal rehabilitation, muscle strengthening.

Copyright (C) 2021 The Author(s): This is an open-access article distributed under the terms of the Creative Commons Attribution 4.0 International License (CC BY-NC 4.0) which permits unrestricted use, distribution, and reproduction in any medium for non-commercial use provided the original author and source are credited.

\section{INTRODUCTION}

The International Continence Society (ICS) defines urinary incontinence (UI) as any involuntary loss of urine that a patient complains about [1]. It is a common affection that affects 25 to $45 \%$ of women in the general population. Its prevalence increases with age until 65 years. There are 3 types of urinary incontinence: stress urinary incontinence (SUI), urge urinary incontinence (UUI) and mixed urinary incontinence (MUI) [2]. Stress urinary incontinence (SUI) characterized by loss of urine that occurs with increased intra-abdominal pressure, such as coughing, laughing, sneezing, jumping, running, lifting, or any other physical activity. The prevalence of SUI in women is $29 \%$ to $75 \%$ depending on age [3].

SUI touches on women's privacy and the notion of cleanliness. It is a recognized factor affecting the quality of life in women $[4,5]$. The age of onset of
UI, type and severity of UI, as well as stress, fears and beliefs are variables that influence the quality of life score significantly [6]. Sexual dysfunction is associated with UI in $25 \%$ to $50 \%$ of cases [7]. Female SUI can be improved by non-invasive medical techniques ranging from simple lifestyle and behavior therapy, to drug therapy and perineal rehabilitation [8]. The latter was introduced in 1948 by the American gynecologist Arnold Kegel [9]. It has become the first-line treatment for stress urinary incontinence due to its safety and the direct and active participation of the patient [8, 10]. This is a non-invasive management that consists of improving SUI through muscle strengthening work, allowing perineal locking at the time of an increase in intra-abdominal pressure to prevent leakage [10]. Several studies and meta-analyzes have addressed the issue of the efficacy of rehabilitative treatment in the treatment of female SUI [11-16]. In some countries, depending on the culture, UI remains a taboo subject that women do not speak about spontaneously and few 
seek medical or even rehabilitative care. This is how we conducted this study to determine the effectiveness of perineal rehabilitation during female SUI.

\section{MATERIALS AND METHODS}

We carried out a prospective, descriptive and mono-centric study, from April to July 2018 in the Department of Physical Medicine and Rehabilitation of the Mohammed VI University of Hospital of Marrakesh.

Ten (10) patients aged from 25 to 65 , referred for perineal rehabilitation were included. They benefited from a rehabilitation protocol performed by the same therapist. We have included: Female subjects over the age of 18 consenting to participate in the study, having a SUI proven by clinical examination (standardized questionnaire, cough test, voiding catalog, postmictional residue) and or exceptionally by a urodynamic assessment.

We did not include: female in immediate postpartum or less than 2 years postpartum, having urinary incontinence by urge, mixed and or of neurological origin, having ATCDs for surgical treatment of urinary incontinence, suffer with a genital prolapse of grade greater than 1 according to the Pelvic Organ Prolapse Quantification (POP-Q) classification and those who developing dysuria and or retention of urine.

During the first session, an initial assessment was made; The latter included an interrogation, a clinical examination with muscle testing of the pelvic floor. During this first meeting, the therapist also explained to the patient the anatomy of the small pelvis using the boards, the mechanism of urinary incontinence, the various risk factors as well as the rules of hygiene of life. Age, parity, duration of lower urinary tract disorders, number of leaks as well as other epidemiological characteristics were collected using a standardized questionnaire. Voluntary muscle contraction had been assessed by manual muscle testing of the pubo coccygeal bundles of elevator muscles of the anus, according to the recommendations of the ICS classified in 4 degrees: Absent, weak, normal and strong. Our rehabilitation protocol required a minimum of 10 sessions of about 40 minutes of exercises depending on the symptoms: Behavioral work, supervised muscle strengthening of the pelvic floor, functional electrostimulation, biofeedback, proprioceptive work and finally behavioral rehabilitation insisting on the good management of the voiding timing, drink control, and relaxation techniques to distract attention. The rehabilitative care began with the awareness of the tone of the perineal muscle. It was initiated by electrostimulation and then reinforced by biofeedback and/or manual labor. We therefore corrected the abdomino-perineal control inversions. The last step consisted of proprioceptive work integrating this contraction into activities of daily living in order to teach the patient to use her perineum when pressure in the abdominal cavity increases and to prevent leaks. We encouraged patients to relay the exercises at home to maintain the results. Assessment of the effect of treatment was based solely on subjective satisfaction.

Variables of the study was: Age, level of education, risk factors for SUI (Parity, menopause, constipation, presence of prolapse), duration of the disorders of the lower urinary tract, the type of protection, the muscle testing of the levator ani, the subjective satisfaction and the number of rehabilitation sessions, performing the urodynamic assessment and rehabilitation technique used.

By statistical analysis we used Microsoft Excel version 2010 for the compilation of the data and the development of the graphs. The mean values and standard deviations were calculated for all the variables studied.

\section{RESULTS}

Ten patients with SUI were able to benefit from perineal rehabilitation. On all the patients, the mean age was $51.1 \pm 13.01$ (range 27 to 65 years), with a mean pregnancy number of $3.62 \pm 1.87$ (range 1 to 8 ) and a mean duration of symptoms of $4.7 \pm 4.71$ (year). Among these patients, $70 \%$ were postmenopausal, 50\% had associated constipation, $60 \%$ had a prolapse and $40 \%$ had performed a urodynamic assessment. All of our patients had vaginal deliveries; none of them played high perineal risk sport. The mean frequency of perineal rehabilitation sessions was $11.58 \pm 3.47$.

The various demographic and clinical variables are shown in figures 1, 2, 3 and 4. The number of sessions and the rehabilitation technique performed, the improvement in muscle testing noted after rehabilitation as well as the subjective efficiency are represented respectively in figures 5, 6, 7 and 8 .

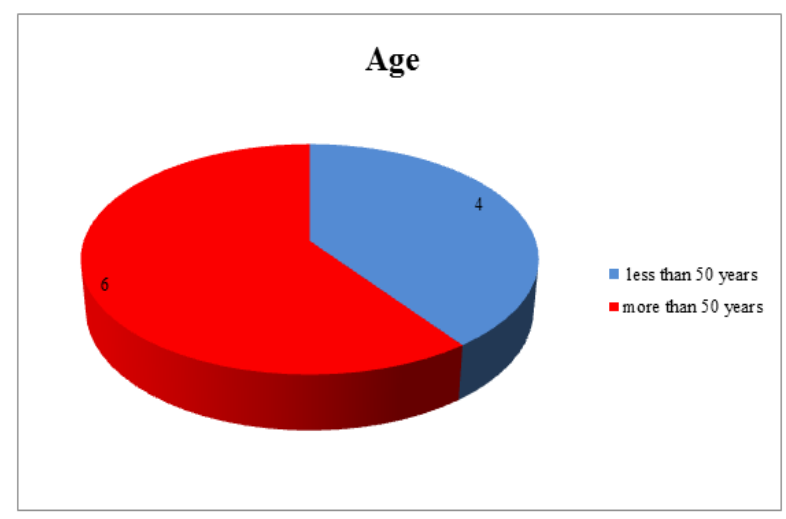

Fig-1: Distribution according to the age 


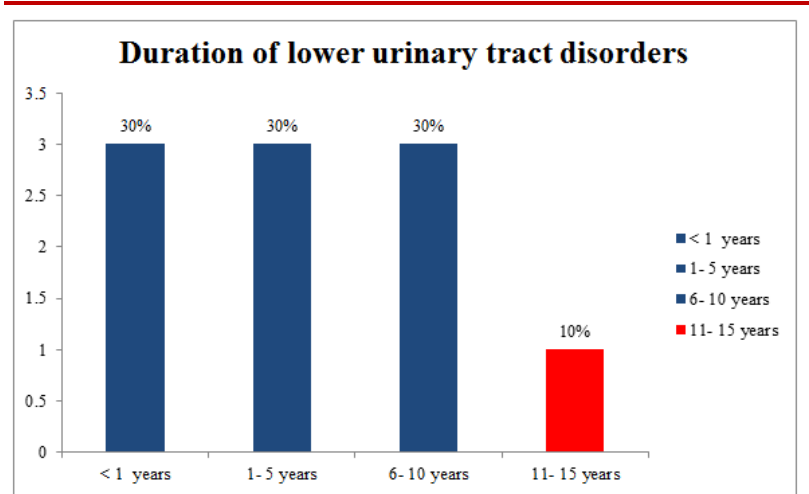

Fig-2: Distribution according to the age of the disorders

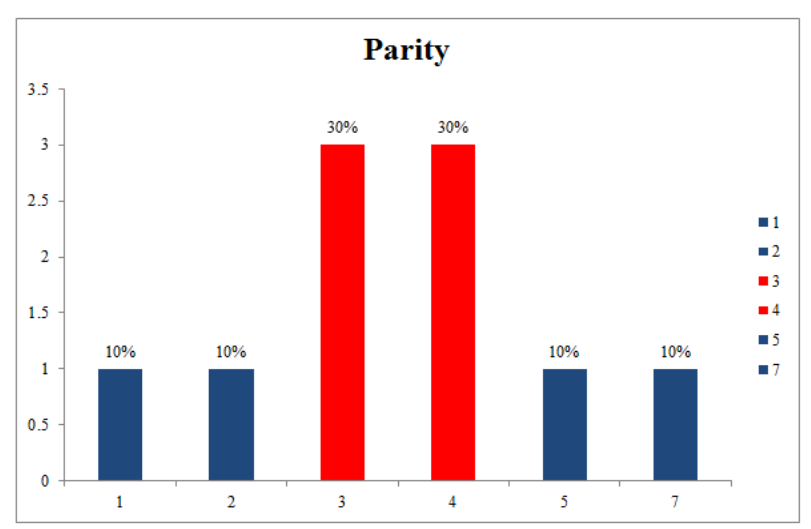

Fig-3: Distribution according to parity

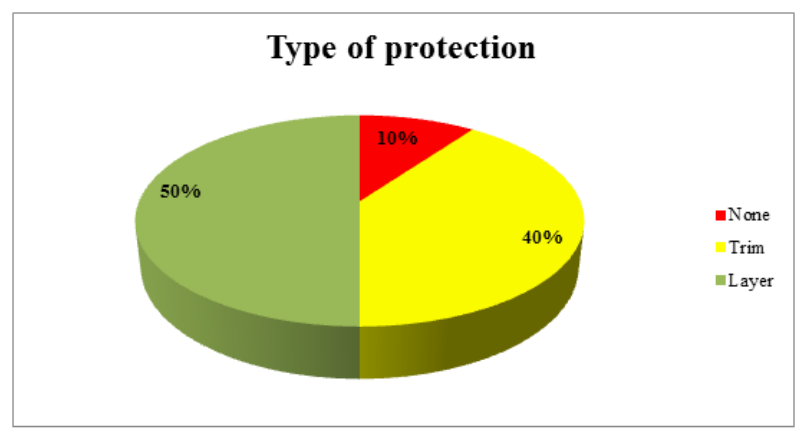

Fig-4: Representation according to the different types of protection used

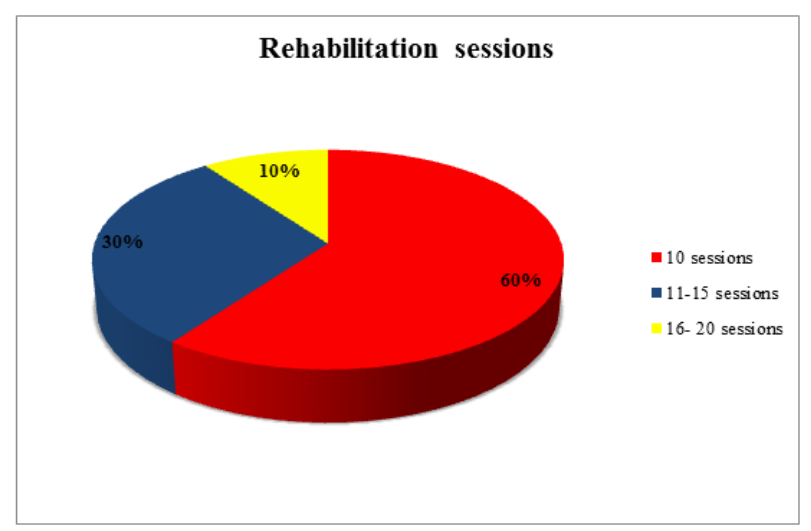

Fig-5: Distribution according to the number of rehabilitation sessions

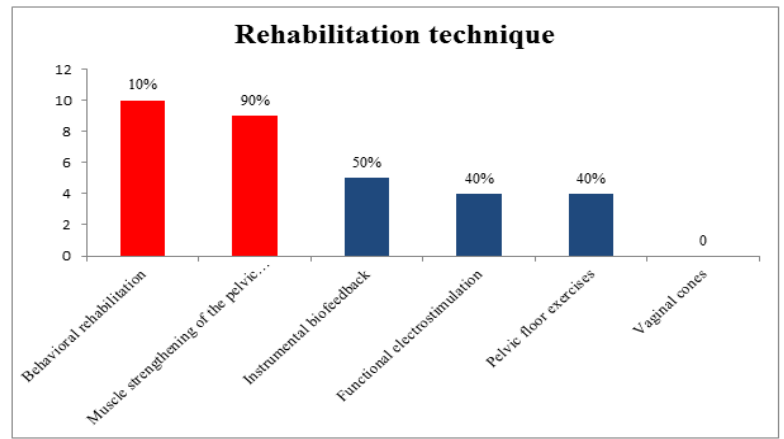

Fig-6: Rehabilitation techniques used

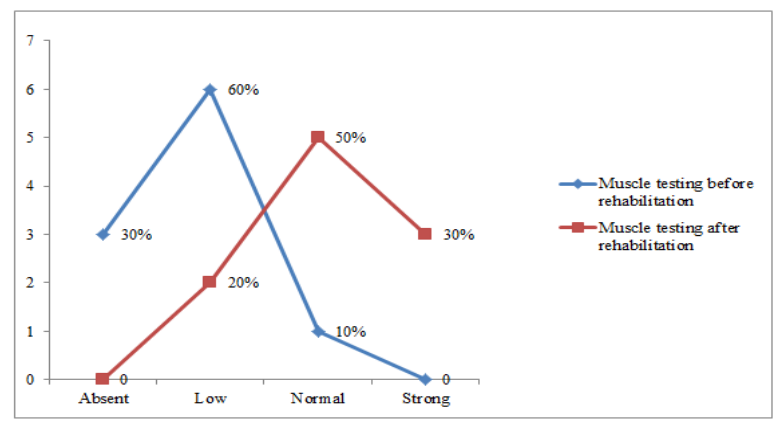

Fig-7: Manual muscle testing of the pubococcygeal bundles of the elevator ani muscles before and after rehabilitation

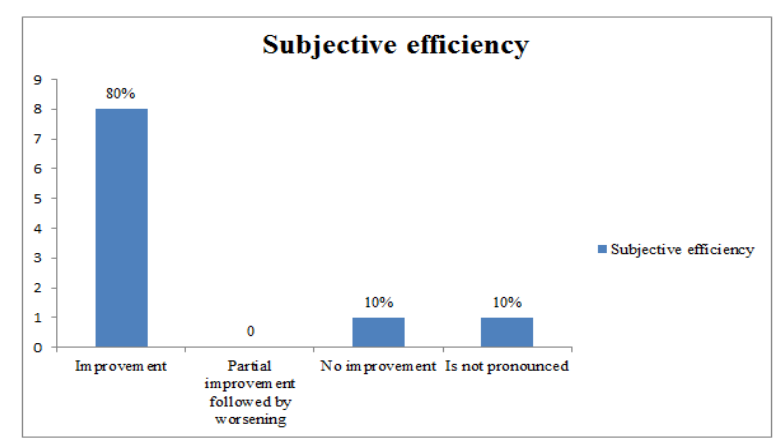

Fig-8: Illustration of subjective efficiency

\section{DISCUSSION}

Our work focused on the role of perineal rehabilitation in female stress urinary incontinence. It should be noted that perineal rehabilitation is a global and multimodal management comprising different facets: a cognitive part (education, awareness of the pelvic floor), a behavioral part of bladder training (modification of voiding habits), a part of muscle strengthening (voluntary contractions against resistance with or without biofeedback and electrostimulation), and also work on posture (balance and position of the pelvis). This rehabilitation has a doublé benefit [16]: On a pure biomedical level by strengthening pelvic muscle tone and more generally by acting on self-image and emotional impact. It is the most prescribed treatment, ahead of medication and surgery. This is the first-line treatment [11,17-19]. Our study population presented the risk factors for SUI described in the literature: More than $50 \%$ were elderly, multiparous and presented with constipation [20]. The late consultation period 
compared to the onset of lower urinary tract disorders proves how much SUI, although having a great impact on quality of life, remains a taboo subject whose admission and demand for care is not systematic.

Depending on the initial assessment performed, different rehabilitation techniques were used in accordance with the recommendations in the literature $[11,17,21,22]$. All of our patients have undergone behavioral rehabilitation although there is still a lot of disagreement about this compared to SUI. Some authors favor muscle strengthening as a first-line treatment [23], which has moreover been shown to be effective in the management of female SUI [13]. We agree with the hypothesis of Thubert $\mathrm{T}$ et al. according to which to expand pelvic-perineal rehabilitation techniques including a cognitive and behavioral approach would appear to be an interesting way [11] and we affirm that behavioral rehabilitation is a factor in the success of treatment.

By using voluntary contraction exercises of the pelvic floor muscles associated with biofeedback (in the event of proprioception disorders) and electrostimulation (in the event of voluntary contraction defect), the rehabilitation allowed an improvement in the tone of these muscles. correlated with a reduction in the frequency of leaks. It is difficult to compare our results with those in the literature due to the heterogeneity of the studies. A systematic review of self-rehabilitation for the treatment of female urinary incontinence showed the effectiveness of home devices: biofeedback (BF) and vaginal cones (VC) used for the treatment of stress urinary incontinence but not their superiority compared to pelvic floor muscle strengthening guided by physiotherapist [13].

Subjective satisfaction fluctuated between 50 and $85 \%$, our results are superimposable on those described by numerous studies [9, 22, 23]. PDudognon et al. believe that the proportion of favorable results (very good and good) does not exceed 54\% at the end of rehabilitation [24].

The long-term benefit of rehabilitation was not evaluated in our study, but the duration of treatment for our patients is close to that described in the majority of studies. Those with the best results had the longest treatment times (3 and 6 months). Overall, the shortterm benefits are well known: $60 \%$ to $77 \%$ of patients experience short-term benefits [25]. Two controlled studies have also shown the short-term effectiveness of rehabilitation on quality of life $[4,6]$. A case-control study using the CONTILIFEL self-questionnaire as judgment criterion, showed that the quality of life of incontinent patients in the group treated by perineal rehabilitation was significantly improved for each parameter tested compared to the untreated group [12]. The long-term benefits have been little studied. The proportion of favorable outcomes undergoes moderate erosion (10\%) over the next 12 to 15 months, and collapses in the course of the 3rd year, not exceeding one in four cases [24]. For patients who continue pelvic floor exercises, the benefit can be maintained at one year, but the failures are significant ( $40 \%$ in one study) and difficult to characterize due to lack of adherence.

\section{CONCLUSION}

Female stress urinary incontinence is a significant symptom due to its large proportion in the population, the social discomfort and the psychological impact it generates.

The objective and subjective effectiveness of perineal rehabilitation makes it the first-line treatment during SUI. The rehabilitative care of SUI is based on pelvic floor strengthening exercise programs that benefit from grade A recommendations. They are more effective when they are intense and are subject to monitoring and supervision by the practitioner. The rehabilitation of SUI has also been the subject of the development of techniques such as biofeedback, electrostimulation, vaginal cones, etc. which have not all shown proof of their effectiveness. It is the physiotherapist's responsibility to choose the most effective and efficient techniques available to him, based on scientific studies evaluating their therapeutic effect while emphasizing the active participation of patients. Based on numerous studies and international recommendations for the rehabilitative management of SUI, we have noted the impact of SUI on improving SUI. Other studies would be necessary to assess the long-term results in order to determine the best parameters (rehabilitation technique, duration, frequency, etc.) to apply.

\section{Contributions of the authors}

All the authors participated in this study, as well as in the writing of this article which they approved.

\section{ABBREVIATIONS}

ICS, International Continence Society; UI, Urinary incontinence; SUI, Stress urinary incontinence; UUI, Urge urinary incontinence; MUI, Mixed urinary incontinence

\section{REFERENCES}

1. Homma, Y. (2003). The standardization of Terminology of Lower Urinary Tract Function: Report from the Standardization Sub-Committee of the International Continence. J. Neurogenic Bladder Soc., 14, 278-289.

2. Faltin, D. L. (2009). Épidémiologie et définition de l'incontinence urinaire féminine. Journal de gynécologie obstétrique et biologie de la reproduction, 38(8), S146-S152.

3. Wood, L. N., \& Anger, J. T. (2014). Urinary incontinence in women. Bmj, 349. 
4. Sinclair, A. J., \& Ramsay, I. N. (2011). The psychosocial impact of urinary incontinence in women. The Obstetrician \& Gynaecologist, 13(3), 143-148.

5. Dragomir, S., Sebag, J., \& Loche, C. M. (2011). Impact de la rééducation périnéale sur la qualité de vie des femmes ayant une incontinence urinaire d'effort ou mixte. Journal de Réadaptation Médicale: Pratique et Formation en Médecine Physique et de Réadaptation, 31(2), 76-80.

6. Kwon, B. E., Kim, G. Y., Son, Y. J., Roh, Y. S., \& You, M. A. (2010). Quality of life of women with urinary incontinence: a systematic literature review. International neurourology journal, 14(3), 133.

7. Avery, J. C., Stocks, N. P., Duggan, P., BraunackMayer, A. J., Taylor, A. W., Goldney, R. D., \& MacLennan, A. H. (2013). Identifying the quality of life effects of urinary incontinence with depression in an Australian population. BMC urology, 13(1), 1-9.

8. Kerdraon, J., \& Denys, P. (2009). Traitement conservateur de l'incontinence urinaire d'effort de la femme. Journal de Gynécologie Obstétrique et Biologie de la reproduction, 38(8), S174-S181.

9. Kegel, A. H. (1948). Progressive resistance exercise in the functional restoration of the perineal muscles. American journal of obstetrics and gynecology, 56(2), 238-248.

10. Leriche, B., \& Conquy, S. (2010). Recommandations pour la prise en charge rééducative de l'incontinence urinaire non neurologique de la femme. Progrčs en Urologie, 20, S104-S108.

11. Thubert, T., Bakker, E., \& Fritel, X. (2015). Pelvic floor muscle training and pelvic floor disorders in women. Gynecologie, obstetrique \& fertilite, 43(5), 389-394.

12. Dragomir, S., Sebag, J., \& Loche, C. M. (2011). Impact de la rééducation périnéale sur la qualité de vie des femmes ayant une incontinence urinaire d'effort ou mixte. Journal de Réadaptation Médicale: Pratique et Formation en Médecine Physique et de Réadaptation, 31(2), 76-80.

13. Kheir, G. B., Grande, P., \& Roumeguère, T. (2020). Auto-rééducation pour le traitement de l'incontinence urinaire féminine: revue de la littérature. Progrès en Urologie.

14. Chamorro, M. V., Casado, J. S., \& García, C. M. (2002). Meta-analysis of the efficacy of perineal rehabilitation for the treatment of female urinary stress incontinence.

15. Fritel, X., Fauconnier, A., Bader, G., Cosson, M., Debodinance, P., Deffieux, X., ... \& Jacquetin, B. (2010). Diagnosis and management of adult female stress urinary incontinence: guidelines for clinical practice from the French College of Gynaecologists and Obstetricians. European Journal of Obstetrics \& Gynecology and Reproductive Biology, 151(1), 1419.
16. Dragomir, S., Sebag, J., \& Loche, C. M. (2011). Impact de la rééducation périnéale sur la qualité de vie des femmes ayant une incontinence urinaire d'effort ou mixte. Journal de Réadaptation Médicale: Pratique et Formation en Médecine Physique et de Réadaptation, 31(2), 76-80.

17. Hermieu, J. F., Denys, P., \& Fritel, X. (2012). Critical review of guidelines for female urinary incontinence diagnosis and treatment. Progres en urologie: journal de l'Association francaise d'urologie et de la Societe francaise d'urologie, 22(11), 636-643.

18. Fritel, X., Fauconnier, A., Bader, G., Cosson, M., Debodinance, P., Deffieux, X., ... \& Jacquetin, B. (2010). Diagnosis and management of adult female stress urinary incontinence: guidelines for clinical practice from the French College of Gynaecologists and Obstetricians. European Journal of Obstetrics \& Gynecology and Reproductive Biology, 151(1), 1419.

19. Thüroff, J. W., Abrams, P., Andersson, K. E., Artibani, W., Chapple, C. R., Drake, M. J., ... \& Tubaro, A. (2011). EAU guidelines on urinary incontinence. Actas Urológicas Españolas (English Edition), 35(7), 373-388.

20. Agence Nationale d'Accréditation et d'Evaluation en Santé. (2003). PEC de l'incontinence urinaire de la femme en médecine générale. Anaes/Service des recommandations professionnelles / mai 2003

21. Dumoulin, C., Hunter, K. F., Moore, K., Bradley, C. S., Burgio, K. L., Hagen, S., ... \& Chambers, T. (2016). Conservative management for female urinary incontinence and pelvic organ prolapse review 2013: Summary of the 5th International Consultation on Incontinence. Neurourology and urodynamics, 35(1), 15-20.

22. Chêne, G., Mansoor, A., Jacquetin, B., Mellier, G., Douvier, S., Sergent, F., ... \& Seffert, P. (2012). Évaluation prospective d'un dispositif de rééducation périnéale à domicile dans l'incontinence urinaire d'effort féminine. Gynécologie obstétrique \& fertilité, 40(6), 350-355.

23. Kegel, A.H. (1949). The physiological traitement of poor tone and function of the genital muscles and of urinary stress incontinence. West J Surg Obstet Gynecol; $57: 527-35$

24. Dudognon, P., Salle, J. Y., Rabiller, M., Milor, E., Munoz, M., Guinvarc'h, S., ... \& Labrousse, C. (1995, January). Rééducation périnéale au cours de l'incontinence urinaire féminine. Résultats à distance. In Annales de réadaptation et de médecine physique (Vol. 38, No. 1, pp. 17-20). Elsevier Masson.

25. Imamura, M., Abrams, P., Bain, C., Buckley, B., Cardozo, L., Cody, J., ... \& Vale, L. (2010). Systematic review and economic modelling of the effectiveness and cost-effectiveness of non-surgical treatments for women with stress urinary incontinence. Health Technology Assessment. 